\title{
Mitral annular plane systolic excursion by cardiac MR is an easy tool for optimized prognosis assessment in ST-elevation myocardial infarction
}

\author{
Agnes Mayr ${ }^{1} \cdot$ Mathias Pamminger ${ }^{1} \cdot$ Martin Reindl $^{2} \cdot$ Simon Greulich $^{3} \cdot$ Sebastian J. Reinstadler ${ }^{2} \cdot$ Christina Tiller $^{2}$. \\ Magdalena Holzknecht ${ }^{2} \cdot$ Timo Nalbach $^{1} \cdot$ David Plappert $^{1} \cdot$ Christof Kranewitter $^{1} \cdot$ Gert Klug $^{2} \cdot$ Bernhard Metzler $^{2}$
}

Received: 29 March 2019 / Revised: 16 July 2019 / Accepted: 24 July 2019 / Published online: 7 August 2019

(C) The Author(s) 2019

\begin{abstract} with ST-elevation myocardial infarction (STEMI) treated with primary percutaneous coronary intervention (pPCI). (MACE) - death, non-fatal myocardial re-infarction, stroke, and new congestive heart failure. $p<0.001)$. conventional LVEF measurement.

\section{Key Points}

- MAPSE determined by CMR independently predicts long-term prognosis following STEMI.

- MACE-free survival is significantly higher in patients with MAPSE $\geq 9 \mathrm{~mm}$ than $<9 \mathrm{~mm}$.

- MAPSE provides significantly higher prognostic implication than conventional LVEF.
\end{abstract}

Objectives The purpose of this study was to assess the comparative prognostic value of mitral annular plane systolic excursion (MAPSE) versus left ventricular ejection fraction (LVEF), measured by cardiac magnetic resonance (CMR) imaging in patients

Methods CMR was performed in 255 STEMI patients within 2 days (interquartile range (IQR) 2-4 days) after infarction. CMR included MAPSE measurement on CINE 4-chamber view. Patients were followed for major adverse cardiovascular events

Results Patients with MACE ( $n=35,14 \%$, median follow-up 3 years [IQR 1-4 years]) showed significantly lower MAPSE ( $8 \mathrm{~mm}[7-8.8]$ vs. $9.6 \mathrm{~mm}[8.1-11.5], p<0.001)$. The association between decreased MAPSE $(<9 \mathrm{~mm}$, optimal cut-off value by c-statistics) remained significant after adjustment for independent clinical and CMR predictors of MACE. The AUC of MAPSE for the prediction of MACE was 0.74 (CI 95\% 0.65-0.82), significantly higher than that of LVEF (0.61 [CI 95\% 0.50-0.71];

Conclusions Reduced long-axis function assessed with MAPSE measurement using CINE CMR independently predicts longterm prognosis following STEMI. Moreover, MAPSE provided significantly higher prognostic implication in comparison with

Keywords Magnetic resonance imaging $\cdot$ ST-elevation myocardial infarction $\cdot$ Prognosis

Agnes Mayr and Mathias Pamminger contributed equally to this work.

Agnes Mayr

agnes.mayr@tirol-kliniken.at

1 University Clinic of Radiology, Medical University Innsbruck, Anichstraße 35, A-6020 Innsbruck, Austria

2 University Clinic of Internal Medicine III, Cardiology and Angiology, Medical University Innsbruck, Anichstraße 35, A-6020 Innsbruck, Austria

3 Department of Cardiology and Cardiovascular Diseases, University Hospital Tübingen, Otfried Müller-Straße 10, 72076 Tübingen, Germany

\begin{tabular}{ll}
\multicolumn{2}{l}{ Abbreviations } \\
AUC & Area under the curve \\
CMR & Cardiac magnetic resonance \\
CoV & Coefficient of variation \\
HR & Hazard ratio \\
ICC & Intraclass correlation coefficients \\
IQR & Interquartile range \\
IS & Infarct size \\
LV & Left ventricular \\
LVEF & Left ventricular ejection fraction \\
MACE & Major adverse cardiac events \\
MAPSE & Mitral annular plane systolic excursion \\
MVO & Microvascular obstruction
\end{tabular}

Abbreviations

AUC Area under the curve

CMR Cardiac magnetic resonance

$\mathrm{CoV}$ Coefficient of variation

HR Hazard ratio

ICC Intraclass correlation coefficients

IQR Interquartile range

IS Infarct size

LV Left ventricular

LVEF Left ventricular ejection fraction

MAPSE Mitral annular plane systolic excursion

Microvascular obstruction 
pPCI Primary percutaneous coronary intervention

SD Standard deviation

SENC Strain-encoded magnetic resonance

STEMI ST-elevation myocardial infarction

\section{Introduction}

Acute myocardial ischemia has a profound impact on myocardial structure and function $[1,2]$. Left ventricular ejection fraction (LVEF) serves as a well-established and robust predictor for worse clinical outcome in patients suffering from acute ST-elevation myocardial infarction (STEMI) [3]. However, propulsion of the intraventricular blood pool is based on a complex interplay of longitudinal shortening, circumferential contraction, and torsion along the long axis of the left ventricle [4]. The main factor for sufficient LV function is ventricular longitudinal shortening, accounting for about $60 \%$ of LV stroke volume both in healthy subjects and in disease, including STEMI [5-7]. Therefore, atrioventricular plane motion has been suggested as an easy-to-measure surrogate for ventricular function and it has been shown that reduced amplitude of valvular plane movement in echocardiography is a predictor of adverse events in patients with various cardiovascular diseases [8]. Impaired mitral annular plane systolic excursion (MAPSE) in cardiac magnetic resonance (CMR) imaging is an independent determinant of all-cause mortality in patients with reduced LVEF $(<50 \%)$, regardless of the underlying cause of impaired systolic function [9]. Rangarajan et al highlighted a decreased MAPSE as determined by CMR to predict major adverse cardiac events (MACE) in a mixed population, including patients with known coronary artery disease or prior myocardial infarction [10]. However, the prognostic implications of CMR-derived MAPSE in a large homogenously treated patient cohort with acute STEMI have not been assessed so far. The aim of this study was therefore to investigate whether CMR-determined MAPSE predicts MACE in patients with reperfused first-time STEMI and to evaluate its prognostic value in comparison with routine LVEF measurement.

\section{Methods}

\section{Study population}

In this prospective observational study, 289 STEMI patients presenting at the coronary care unit of the Innsbruck University Hospital (Innsbruck, Austria) were initially included. After exclusion of 34 patients, a final cohort of 255 STEMI patients was analyzed. Reasons for exclusion were missing LVEF measurements due to limited image quality caused by arrhythmia and breathing artifacts $(n=10)$ or incomplete short-axis cine coverage of the LV image stack $(n=3)$, inadequate imaging plane for septal MAPSE measurements due to inclusion of the LV outflow tract or aortic valve with subsequent invisibility of the septal insertion point of the mitral valve $(n=9)$, lack of follow-up accessibility because of a changed telephone number or withdrawal of consent to further data acquisition $(n=11)$ or refusion of contrast agent application $(n=1)$.

The following inclusion criteria were applied: first STEMI according to the redefined European Society of Cardiology/ American College of Cardiology committee criteria [11] revascularization by primary percutaneous coronary intervention (pPCI) within $24 \mathrm{~h}$ after the onset of symptoms, an estimated glomerular filtration rate $>30 \mathrm{~mL} / \mathrm{min}$ per $1.73 \mathrm{~m}^{2}$, and Killip class $<3$ at time of CMR. Exclusion criteria were age $<18$ years, any history of previous myocardial infarction or coronary intervention, and any contraindication to CMR examination (pacemaker, claustrophobia, orbital foreign body, cerebral aneurysm clip, or known or suggested contrast agent allergy to gadolinium).

The clinical endpoint of the present study was the occurrence of MACE defined as a composite of all-cause death, non-fatal myocardial re-infarction, stroke, and congestive heart failure. Re-infarction was defined in accord with the redefined European Society of Cardiology/American College of Cardiology committee [11] and new congestive heart failure was defined as the first episode of cardiac decompensation requiring diuretic therapy. Follow-up data for clinical endpoint assessment were collected at 6 months and 12 months after STEMI then annually via telephone interview using a standardized questionnaire [12]. All interviews were performed by trained personnel blinded to baseline CMR, laboratory, and angiographic findings. The declared endpoints were checked afterwards by carefully reviewing the corresponding medical records.

Before inclusion in the present study, all participants gave their written informed consent. The study was approved by the local research ethics committee and conducted in conformity with the Declaration of Helsinki.

\section{Cardiac magnetic resonance imaging}

All patients were investigated on a clinical 1.5-T MR imaging unit (AVANTO_fit; Siemens Healthineers) within 2 days (interquartile range (IQR) 2-4 days) after successfully reperfused first acute STEMI by pPCI. The standardized imaging protocol of our research group was published in detail previously [13]. Briefly, LV volumes and function were assessed on short-axis cine images using breath-hold, retrospective ECG-triggered trueFISP bright-blood sequences. For postprocessing, standard software (ARGUS; Siemens) was applied. Papillary muscles were assigned to the LV volume [14]. 
End-diastolic and end-systolic mitral annular planes were defined on a long-axis four-chamber view by connecting the septal and lateral attachment of the mitral valve to the myocardium on the respective images of the long-axis stack, with end-diastole being defined by the largest diameter of the left ventricle and end-systole as the image immediately before mitral valve opening. "Septal" MAPSE was defined as the perpendicular distance of the end-systolic mitral annular plane to the end-diastolic plane, measured in regard to the septal attachment of the mitral valve in end-diastole as shown in Fig. 1. Similarly, "lateral" MAPSE was defined as the distance of the lateral attachment of the mitral valve between enddiastole and end-systole. Average MAPSE is the calculated mean between septal and lateral MAPSE.

Late gadolinium enhancement images were acquired $15 \mathrm{~min}$ after the application of a $0.2 \mathrm{mmol} / \mathrm{kg}$ bolus of contrast agent (Multihance; Bracco) using an ECGtriggered phase-sensitive inversion recovery sequence with consecutive short-axis slices. The late gadolinium enhancement extent of each slice was quantified by using a PACS workstation (IMPAX; Agfa HealthCare). We defined "hyperenhancement" as +5 SDs above the signal intensity of remote myocardium in the opposite myocardial segment of the LV [15]. Infarct size (IS) was expressed as a percentage of LV myocardial mass. Microvascular obstruction (MVO) was defined as a persisting area of "hypoenhancement" within the infarcted, hyperenhanced territory [16]. All CMR images were analyzed by experienced observers, blinded to clinical and angiographic data. A randomly determined sample of 30 study participants was evaluated three times to evaluate intra-observer and inter-observer variability.

\section{Statistical analysis}

SPSS Statistics (version 24.0; IBM Corp) and MedCalc (Version 15.8; MedCalc Software bvba) were used for statistical analyses. According to the presence or absence of normal distribution, continuous variables are presented as mean $\pm \mathrm{SD}$ or median with corresponding IQR. Categorical variables are expressed as absolute numbers and percentages. The differences in continuous variables between 2 groups were evaluated by the Mann-Whitney $\mathrm{U}$ test or Student $t$ test, as appropriate. The chi-square test was used to assess the differences in categorical variables. Spearman test was applied to calculate correlations of continuous variables. All parameters included in Table 1 were included in univariable Cox regression analyses. Variables with a $p<0.10$ in univariable analysis and age were entered in a multivariable model [12]. Two different multivariate models were compiled to ensure statistical robustness with respect to our sample size and event rate. In
Fig. 1 End-diastolic (solid yellow line) and end-systolic mitral annular plane (red line) were defined on a long-axis four-chamber view by connecting the septal and lateral attachment of the mitral valve to the myocardium on the respective images of the long-axis stack, with end-diastole being defined by the largest diameter of the left ventricle and end-systole as the image immediately before mitral valve opening. MAPSE (dotted yellow line) was defined as the perpendicular distance of the end-systolic mitral annular plane to the end-diastolic plane, measured in regard to the septal attachment of the mitral valve in end-diastole

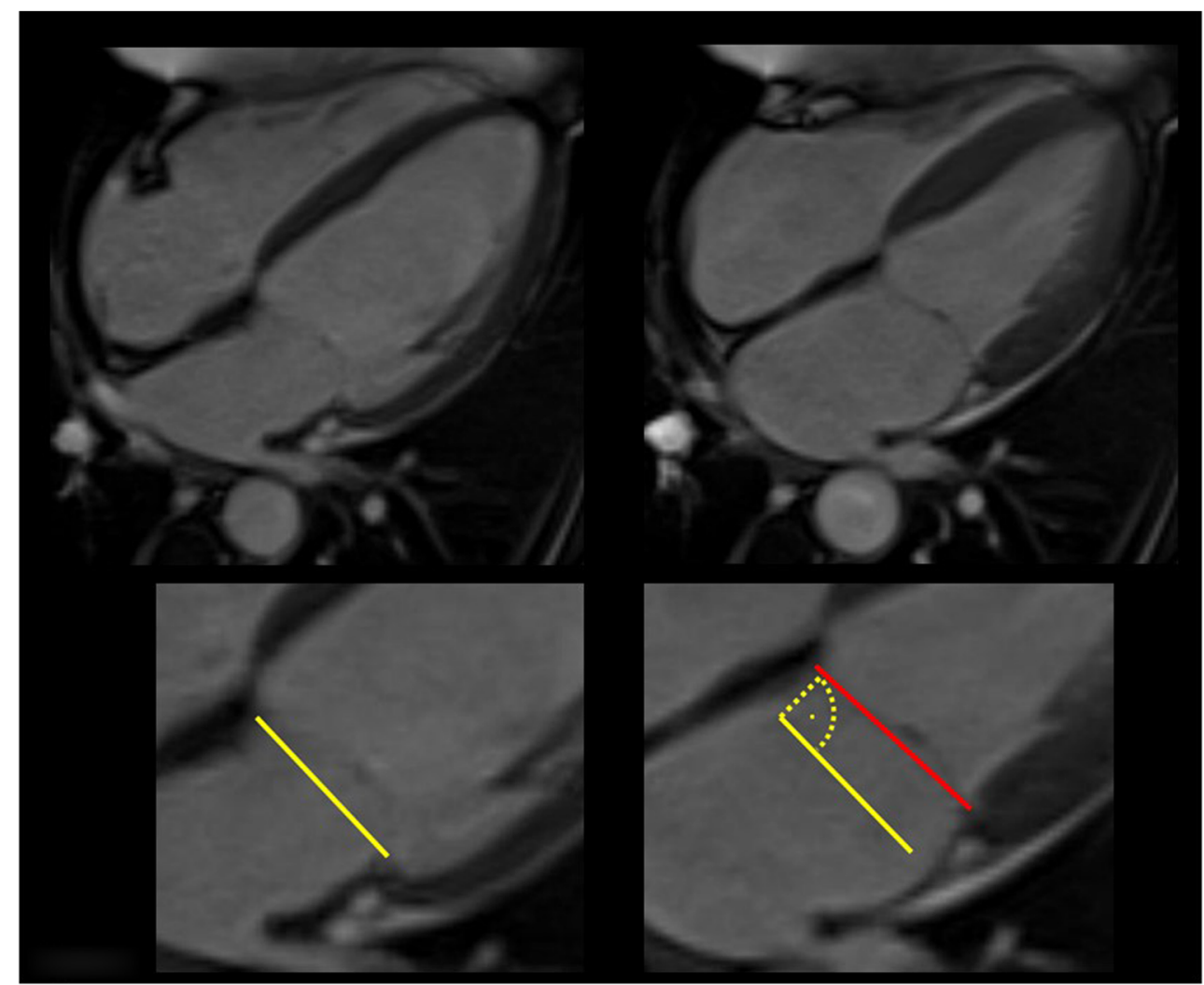


Table 1 Patients characteristics

\begin{tabular}{|c|c|c|c|c|}
\hline & $\begin{array}{l}\text { Total population } \\
(n=255)\end{array}$ & $\begin{array}{l}\text { MACE } \\
(n=35,13.7 \%)\end{array}$ & $\begin{array}{l}\text { No MACE } \\
(n=220,86.3 \%)\end{array}$ & $p$ value \\
\hline Age, years & $57( \pm 11)$ & $61.7( \pm 11)$ & $56( \pm 11)$ & 0.01 \\
\hline Female, $n(\%)$ & $40(15.7)$ & $6(17.1)$ & $34(15.5)$ & 0.80 \\
\hline Body mass index $\left(\mathrm{kg} / \mathrm{m}^{2}\right)$ & $26.1[24.4-28.3]$ & $26[24-28]$ & $26.1[24.4-28.4]$ & 0.76 \\
\hline Diabetes, $n(\%)$ & $21(8.2)$ & $5(14.3)$ & $16(7.3)$ & 0.18 \\
\hline Hyperlipidemia, $n(\%)$ & $156(61.2)$ & $25(71.4)$ & $131(59.5)$ & 0.20 \\
\hline Smoking, $n(\%)$ & $136(53.3)$ & $15(42.9)$ & $121(55)$ & 0.27 \\
\hline Hypertension, $n(\%)$ & $139(54.5)$ & $25(71.4)$ & $114(51.8)$ & 0.04 \\
\hline Positive family history, $n(\%)$ & $73(28.6)$ & $7(20)$ & $66(30)$ & 0.31 \\
\hline Peak hs-cTnT (ng/L) & 3594 [275-6884] & 4257 [23.5-10166] & 3471 [298.3-6672] & 0.27 \\
\hline $\operatorname{LVEF}(\%)$ & $54.4[48-59.6]$ & $51.1[42.2-57.3]$ & $54.5[48.8-59.7]$ & 0.05 \\
\hline LVEDV (mL) & 147.9 [120.9-167.6] & 153.5 [115.8-166.9] & 147.1 [121.4-168.9] & 0.81 \\
\hline LVESV (mL) & $67.8[50-82.5]$ & $70.5[52.9-98.2]$ & $67.2[50-81.7]$ & 0.28 \\
\hline LV mass (g) & $136.7[115.5-157.6]$ & $143.3[122.5-160.2]$ & $136.5[115.4-156.3]$ & 0.38 \\
\hline Septal MAPSE (mm) & $9.4[7.9-11.3]$ & $8[7-8.8]$ & $9.6[8.1-11.5]$ & $<0.001$ \\
\hline Lateral MAPSE (mm) & $11.1[9.2-13.1]$ & $9.9[7.6-11.5]$ & $11.4[9.4-13.2]$ & 0.002 \\
\hline Average MAPSE (mm) & $10.2[8.6-12]$ & $8.9[7.4-10.1]$ & $10.5[8.9-12.2]$ & $<0.001$ \\
\hline IS, $\%$ of LVMM & $16.9[7.3-23.8]$ & $15.9[10.6-21.8]$ & $13.5[6.9-24]$ & 0.32 \\
\hline MVO, $n(\%)$ & $131(51.4)$ & $23(65.7)$ & $108(49.1)$ & 0.07 \\
\hline Infarct localization & & & & 0.04 \\
\hline Anterior (LAD) & $118(46.3)$ & $22(62.9)$ & $96(43.6)$ & \\
\hline Non-anterior (RCA and LCX) & $137(53.7)$ & $13(34.1)$ & $124(56.4)$ & \\
\hline
\end{tabular}

hs-cTnT, high-sensitivity cardiac troponin T; LVEF, left ventricular ejection fraction; LVEDV, left ventricular end-diastolic volume; LVESV, left ventricular end-systolic volume; MAPSE, mitral annular plane systolic excursion; IS \% of LVMM, infarct size in percent of left ventricular myocardial mass; MVO, microvascular obstruction; LAD, left anterior descending artery; RCA, right coronary artery; LCX, left circumflex artery

addition to the multivariable model comprising continuous variables (raw data), we formed a dichotomized model including the variables dichotomized according to the optimal cut-off determined by C-statistics to warrant an accurate comparison of hazard ratios. Together with MAPSE, age, the presence of hypertension, and infarct localization were entered in a model of clinical risk factors. Likewise, the presence of MVO and LVEF together with MAPSE were included in a model of imaging risk factors. Area under the curve (AUC) differences were appraised by a method established by de Long et al [17]; accordantly, the AUC values were interpreted using the following standard categories: negligible $(\leq 0.55)$, small $(0.56-0.63)$, moderate $(0.64-0.70)$, and strong $(\geq 0.71)$.

MACE-free survival was estimated and depicted by the Kaplan-Meier method, and differences were assessed by the log-rank test.

Intra-observer and inter-observer variabilities of MAPSE measurements were determined by intraclass correlation coefficients (ICC) and coefficients of variation (CoV). A $p$ value of $<0.05$ was defined as statistically significant.

\section{Results}

\section{Subject characteristics}

We included 255 consecutive STEMI patients with a total ischemia time of 205 (IQR, 132-351) minutes. Mean age of the overall population was $57( \pm 11)$ years. Baseline characteristics and CMR parameters of the overall cohort are listed in Table 1.

The median of septal MAPSE was $9.4 \mathrm{~mm}$ (IQR 7.9$11.3 \mathrm{~mm}$ ) and median LVEF was $54.4 \%$ (IQR 48-59.6\%). Acute IS in \% of LV myocardial mass was $16.9 \%$ (IQR 7.323.8\%) and MVO was detected in 131 patients (51.4\%).

\section{Determinants of MACE}

Thirty-five (13.7\%) patients experienced a MACE event (8 deaths, 14 myocardial re-infarctions, 8 strokes, 5 congestive heart failures). Median follow-up time was 3 years (IQR 14 years). The median time to event was 36 months (IQR 12 53 months). Table 1 provides all parameters separately for 
patients with $(n=35 ; 14 \%)$ and without $(n=220 ; 86.3 \%)$ MACE. There was a significant association of age $(p=0.01)$ and the incidence of hypertension $(p=0.04)$ as well as infarct localization $(p=0.04)$ with the occurrence of MACE. Regarding CMR parameters, patients displaying MACE showed lower LVEF $(p=0.05)$ and MAPSE $(p<0.001)$ and displayed a trend to a higher incidence of MVO $(p=0.07)$. No significant association of MACE with other CMR parameters (IS, LV end-diastolic volume, and LV end-systolic volume) or clinical parameters (diabetes, hyperlipidemia, smoking, positive family history, peak high-sensitivity cardiac troponin T, and infarct-related artery) were found (all $p>0.05$ ). In multivariable analysis, MAPSE remained a significant independent predictor of MACE, in both clinical risk factor model (hazard ratio $=0.77$ [CI 95\% 0.66-0.90]; $p=0.001$ ) and CMR risk factor model (hazard ratio $=0.83$; CI 95\% $0.73-0.95$, $p<0.006$ ), see Table 2 .

\section{Prognostic value of MAPSE}

After adjustment for both clinical and imaging risk factors that were univariate predictors $(p<0.10)$, MAPSE $<9 \mathrm{~mm}$ remained a significant predictor of MACE in the model of dichotomized clinical risk factors (hazard ratio $=6.02$; CI $95 \% 2.47-14.69, p<0.001)$ as well as in the model of dichotomized imaging risk factors (hazard ratio $=5.03$; CI $95 \%$ 2.11-12.01, $p<0.001)$, see Table 3 .

Receiver operating characteristics (ROC) analysis revealed significantly higher $(p=0.03)$ AUC of septal MAPSE $(0.74$ [95\% CI 0.66-0.82]) compared with average MAPSE $(0.70$ [95\% CI 0.61-0.79]) as well as in comparison with lateral MAPSE (0.66, [95\% CI 0.57-0.75], $p=0.01$ ) for the prediction of MACE, see Fig. 2a.

The optimal cut-off value of septal MAPSE was $9 \mathrm{~mm}$ providing the highest sensitivity (80\%) and specificity $(63 \%)$ (Fig. 2b). This AUC of MAPSE was significantly higher ( $p=$
$0.03)$ than the AUC of LVEF (0.60 [95\% CI $0.50-0.78]$ ) for the prediction of MACE.

According to the Kaplan-Meier analysis, patients with MAPSE $<9 \mathrm{~mm}$ showed a significantly lower MACE-free survival $(p=0.001)$ than patients with MAPSE $\geq 9 \mathrm{~mm}$ (Fig. 3).

\section{Intra-observer and inter-observer variability for MAPSE measurements}

Intra-observer analyses of septal MAPSE measurements showed an excellent agreement with an ICC of 0.94 (95\%CI $0.75-0.98)$. The coefficient of variability was $13 \%$. Similarly, inter-observer analyses showed excellent reproducibility of 0.89 (95\% CI $0.50-0.98)$ and coefficient of variability $16 \%$. The mean of the absolute difference between the two reader measurements was $0.57 \mathrm{~mm}$. The mean of the absolute difference between the main reader measurements at different time points was $0.38 \mathrm{~mm}$.

\section{Discussion}

This study is the first to evaluate the prognostic value of CMR-derived MAPSE in a large STEMI cohort treated by pPCI. The main study findings can be summarized as follows:

1. Septal MAPSE determined by CMR early after reperfused STEMI is a powerful, independent predictor of MACE at long-term follow-up (3 years).

2. Patients with MAPSE $\geq 9 \mathrm{~mm}$ showed a significantly higher MACE-free survival than patients with MAPSE $<9 \mathrm{~mm}$.

3. The predictive value of MAPSE was significantly higher than that of conventional LVEF.
Table 2 Cox regression analysis for the prediction of MACE

\begin{tabular}{llcll}
\hline & $\begin{array}{l}\text { Univariable analysis } \\
\text { Hazard ratio }(95 \% \mathrm{CI})\end{array}$ & $p$ value & $\begin{array}{l}\text { Multivariable analysis } \\
\text { Hazard ratio (95\% CI) }\end{array}$ & $p$ value \\
\hline Clinical risk factor model & & & & \\
Age & $1.021(0.989-1.054)$ & 0.19 & - & - \\
Hypertension & $1.410(0.660-3.014)$ & 0.38 & - & - \\
Infarct localization & $2.283(1.137-4.585)$ & 0.02 & - & 0.001 \\
MAPSE & $0.803(0.706-0.914)$ & $<0.001$ & $0.770(0.658-0.901)$ & - \\
CMR risk factor model & & & & - \\
MVO & $1.880(0.935-3.782)$ & 0.07 & - & - \\
LVEF & $0.950(0.917-0.985)$ & 0.05 & - & 0.006 \\
MAPSE & $0.796(0.710-0.893)$ & $<0.001$ & $0.829(0.726-0.947)$ & \\
\hline
\end{tabular}

CI, confidence interval; MAPSE, mitral annular plane systolic excursion; CMR, cardiac magnetic resonance; MVO, microvascular obstruction; LVEF, left ventricular ejection fraction 
Table 3 Cox regression analysis for the prediction of MACE (dichotomized clinical and CMR risk factors)

\begin{tabular}{llcll}
\hline & $\begin{array}{l}\text { Univariable analysis } \\
\text { Hazard ratio }(95 \% \mathrm{CI})\end{array}$ & $p$ value & $\begin{array}{l}\text { Multivariable analysis } \\
\text { Hazard ratio }(95 \% \mathrm{CI})\end{array}$ & $p$ value \\
\hline Clinical risk factor model & & & & - \\
Age $>60$ & $1.338(0.674-2.659)$ & 0.41 & - & - \\
Hypertension & $1.537(0.719-3.287)$ & 0.27 & - & - \\
Infarct localization & $1.805(0.899-3.622)$ & 0.10 & - & $<0.001$ \\
MAPSE $<9$ mm & $5.056(2.182-11.716)$ & $<0.001$ & $6.021(2.469-14.682)$ & - \\
CMR risk factor model & & & & - \\
MVO & $1.880(0.935-3.782)$ & 0.08 & - & - \\
LVEF $<52 \%$ & $2.360(1.209-4.608)$ & 0.01 & - & $5.030(2.108-12.003)$ \\
MAPSE $<9$ mm & $5.947(2.595-13.630)$ & $<0.001$ & 501 \\
\hline
\end{tabular}

CI, confidence interval; MAPSE, mitral annular plane systolic excursion; CMR, cardiac magnetic resonance; MVO, microvascular obstruction; LVEF, left ventricular ejection fraction
Therefore, these results argue for the routine assessment of MAPSE in patients early after acute STEMI.

The longitudinal function of the LV is the main contributor to left ventricular pump function [6, 7]. Supposed longitudinal orientation of subendocardial myocardial fibers has long been discussed as a cause for impaired longitudinal function in patients with acute myocardial infarction [5, 18-20]. However, recent studies on myocardial microstructure assessed by CMR diffusion tensor imaging reported a spiral configuration of myocytes gradually shifting from a righthand helix subendocardially to a left-hand helix subepicardially, working as a syncytium [21]. In myocardial infarction, myocardium adjacent to the scarred area shows a decrease in the right-hand helix of subendocardial fibers as

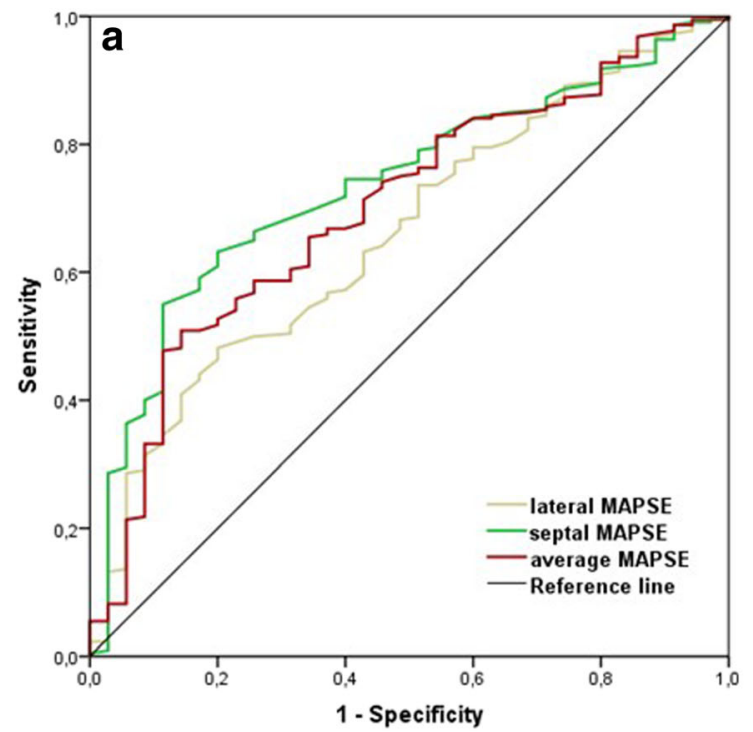

Fig. 2 a ROC analysis of septal MAPSE (AUC 0.74 [95\% CI $0.66-$ $0.82]$ ), average MAPSE (AUC 0.70 [95\% CI 0.61-0.79]), and lateral MAPSE (AUC 0.66, [95\% CI 0.57-0.75]) for the prediction of MACE. AUC indicates area under the curve; MAPSE, mitral annular plane systolic excursion; ROC, receiver operating characteristics. b ROC well as a decreased range of helix angles across the whole myocardial wall in subacute myocardial infarction in a primate model [22]. Additionally, myocardial microstructures termed "sheetlets," consisting of several myocytes, work together as mechanical units to change tilting angle over the cardiac cycle and are thought to be accountable for systolic myocardial thickening [21]. However, possible changes of sheetlet mechanics in myocardial infarction have not been studied yet. Therefore, underlying mechanisms of impaired longitudinal function in myocardial infarction are still to be discussed, as causality between myocardial fiber architecture and cardiac function has not been proven yet [23].

However, global longitudinal function remains the main contributor to LVEF in ischemic heart disease [5]. MAPSE

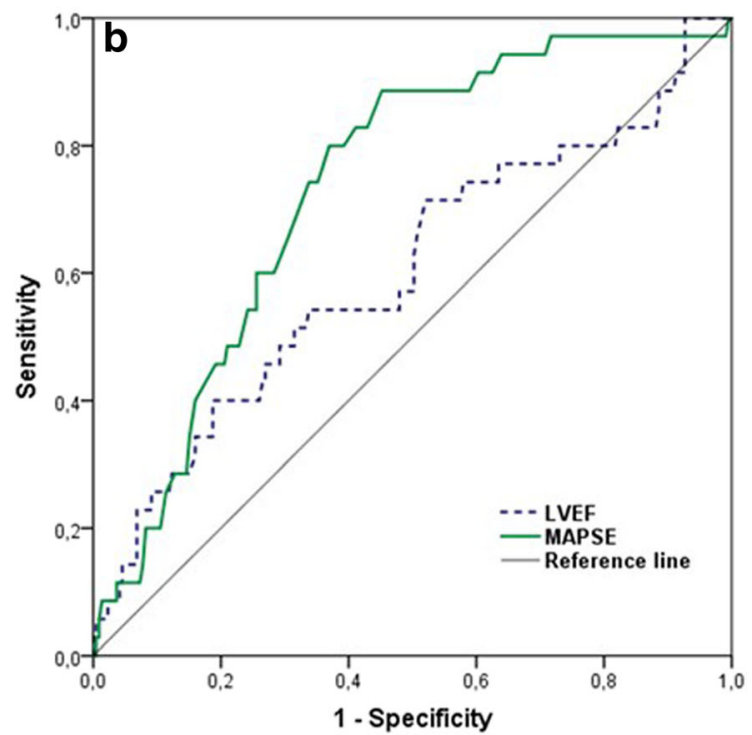

analysis of MAPSE (AUC 0.74 [95\% CI 0.66-0.82]) and LVEF (AUC 0.60 , [95\% CI $0.50-0.78]$ ) for the prediction of MACE. AUC, area under the curve; LVEF, left ventricular ejection fraction; MAPSE, mitral annular plane systolic excursion; ROC, receiver operating characteristics 


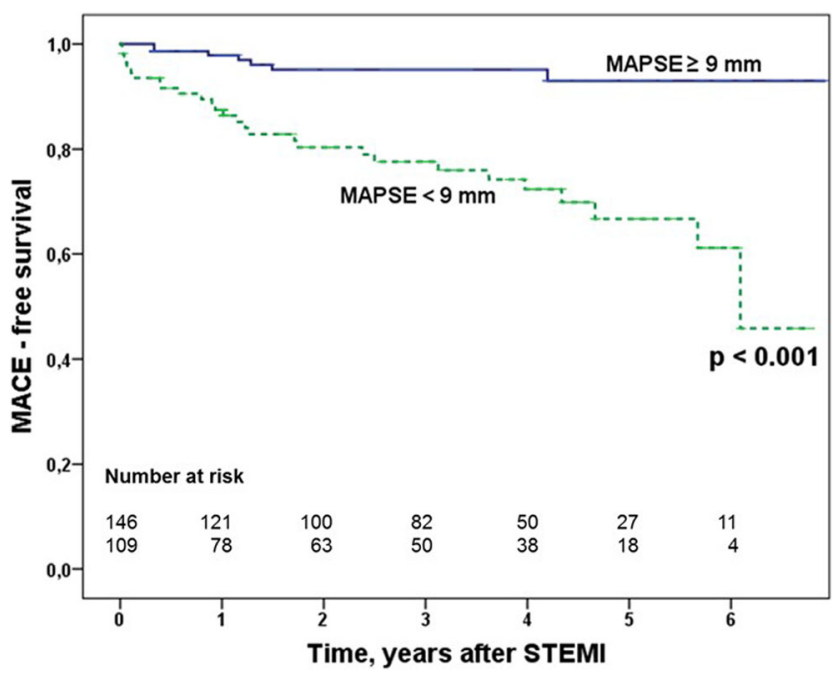

Fig. 3 Kaplan-Meier curves for the occurrence of MACE stratified by $\geq$ and $<9 \mathrm{~mm}$. MAPSE was calculated by ROC analysis. MACE indicates major adverse cardiovascular events; MAPSE, mitral annular plane systolic excursion; ROC, receiver operating characteristics

as recorded by different echocardiographic methods, including M-mode, tissue Doppler, and strain imaging and speckle tracking assessed in both acute and chronic setting of myocardial infarction, was shown to predict adverse events [18-20, 24]. However, although easily available and cost-efficient, these techniques suffer from several disadvantages such as angle dependency issues for M-mode method or problems that are related to signal noise [25]. Strain imaging overcomes monodimensional focality by providing segmental and global information regarding longitudinal deformation but is highly dependent on imaging quality and operator experience [26]. Advanced CMR tools provide incremental prognostic stratification in patients with STEMI $[27,28]$ and easy-to-measure CMR-derived MAPSE has been linked to adverse outcome after STEMI. However, prognostic data of MAPSE in CMR either come from large all-comers cohorts without specific heart disease or from smaller groups of patients with myocardial infarction [10, 29, 30]. Our homogeneously treated a large patient group with first acute STEMI septal MAPSE with an optimal cut-off value of $9 \mathrm{~mm}$ provided the highest value for MACE prediction. Furthermore, septal MAPSE was shown to provide significantly higher predictive value after STEMI compared with lateral or average MAPSE. Other CMR studies revealed threshold values for lateral MAPSE of $9 \mathrm{~mm}$ [9] and $11 \mathrm{~mm}$ [10] respectively as cut-off points for risk stratification in mixed CMR populations. Lateral mitral annulus movement in healthy subjects is usually greater than septal movement (16 $\pm 3 \mathrm{~mm}$ compared with $13 \pm 3 \mathrm{~mm}$ ) [31]. Therefore, a larger cut-off value for lateral MAPSE is in accordance with anatomical properties. Romano et al used death as a primary endpoint, which might serve as an explanation why their reported cut-off for lateral MAPSE $(9 \mathrm{~mm})$ is equal as we found for septal MAPSE. However, Pahlm et al reported a decrease in global and regional MAPSE in infarcted as well as remote myocardium, supporting our thesis that septal MAPSE provides prognostic information regardless of infarct localization [32]. Based on the significantly higher predictive value of septal MAPSE compared with lateral and average MAPSE in our population as well as on the independency of MAPSE decrease from infarct location, we consider the exclusive measurement of septal MAPSE to be reasonable.

Numerous studies have demonstrated LVEF as a marker of global systolic myocardial function and a powerful predictor of morbidity and mortality in patients with acute reperfused myocardial infarction $[33,34]$. Recently presented CMR indices and scores integrating several structural and/or morphological variables were suggested to provide incremental prognostic validity in STEMI patients [27, 35]. However, septal MAPSE is a unique, simple measurable and effective marker in standard CMR and our study showed that it offers prognostic information that adds beyond traditional clinical and imaging cardiac risk factors. Moreover, the high reproducibility of MAPSE measurements shown by Romano et al was confirmed in our study by a very low intra- and inter-observer variability [29]. The superior prognostic performance of MAPSE compared with that of LVEF in STEMI patients may be explained by the suspicion that longitudinally running myocardial fibers are located subendocardially and are most affected by ischemia. However, LVEF primarily tracks radial function of the myocardium. Anyhow, recent studies suggest more complex organization of cardiomyocyte microstructure and dynamics [21]. In accordance with the findings previously reported by van Kranenburg et al, infarct size in our study was not significantly associated with MACE occurrence [36]. To what extent large but only subendocardial infarcts are less favorable for long-axis function and prognosis than smaller but transmural infarcts remains to be investigated.

Despite rapid reperfusion of epicardial coronary circulation by pPCI in STEMI, severe microvascular dysfunction, related to initial ischemia and/or to reperfusion injury, may persist [37]. MVO is a significant and independent short- and longterm prognosticator for morbidity and mortality after STEMI $[38,39]$. In our current study, patients with MVO showed a trend towards higher incidence of MACE but did not reach significance $(p=0.07)$. One explanation could be that our definition of adverse outcome included stroke (23\% of total MACE). Previous studies only included death and cardiac events (re-infarction, hospitalization for heart failure) [36, 38].

Specialized CMR techniques such as strain-encoded MR (SENC) [39] and feature-tracking software promise risk stratification in patients with various cardiac diseases [28, 40-45]. Several studies recently assessed the relationship of LV strain, infarct characteristics such as edema or hemorrhage, and their association with prognosis in patients with acute myocardial infarction; while Eitel et al [45] highlighted CMR feature tracking to have incremental prognostic value above LVEF 
and infarct size, others [41] showed tissue tracking to not substantially improve risk reclassification beyond LVEF, infarct size, and MVO. However, this technique requires the use of specialized software and consequently has not achieved widespread clinical use [46]. Moreover, strain imaging is difficult to measure in the acute setting, and there is a lack of evidence for its benefit. Good image quality is an essential prerequisite for strain analysis as well as for LVEF measurement while in case of poor imaging quality, MAPSE could still be a suitable choice for assessing longitudinal function.

Septal MAPSE is simple to measure on 4-chamber routine cine images, available from any vendor without any specific software and with a good inter- and intra-observer variability.

\section{Limitations}

Due to our inclusion criteria, the results of this study only apply to a selected patient group and must not be generalized for patients with recurrent myocardial infarction or other cardiac pathologies that entail left ventricular remodeling. However, with an overall incidence rate of STEMI of 43 to 144 per 100,000 in Europe [47], specific data for this particular group should be available to support clinical decision-making.

We did not measure atrial volumes or left atrial functional parameters for the prediction of outcome after STEMI as it was suggested before [48]. Future studies may address the left atrium parameters. We only measured absolute values of septal MAPSE and did not take into account factors like patient size, ventricular diameters, sex, or age that might influence absolute mitral plane motion [49, 50]. Upcoming technologies such as SENC imaging and new feature-tracking software have not been evaluated. However, their clinical relevance is still controversial [41].

\section{Conclusion}

We provide a parameter that is simple to measure and easy to implement in clinical routine without the need for specialized devices or software. Reduced septal MAPSE $<9 \mathrm{~mm}-$ assessed by cine $\mathrm{CMR}$ - is an independent long-term predictor of MACE in patients after first-time STEMI undergoing pPCI. It provides superior prognostic value compared with LVEF.

Funding Open access funding provided by University of Innsbruck and Medical University of Innsbruck. The authors state that this work has not received any funding.

\section{Compliance with ethical standards}

Guarantor The scientific guarantor of this publication is Agnes Mayr, MD.
Conflict of interest The authors of this manuscript declare no relationships with any companies whose products or services may be related to the subject matter of the article.

Statistics and biometry No complex statistical methods were necessary for this paper.

Informed consent Written informed consent was obtained from all subjects (patients) in this study.

Ethical approval Institutional Review Board approval was obtained.

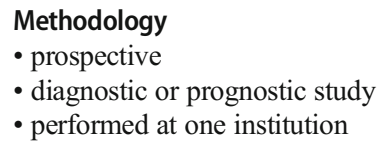

Open Access This article is distributed under the terms of the Creative Commons Attribution 4.0 International License (http:// creativecommons.org/licenses/by/4.0/), which permits unrestricted use, distribution, and reproduction in any medium, provided you give appropriate credit to the original author(s) and the source, provide a link to the Creative Commons license, and indicate if changes were made.

\section{References}

1. Jennings RB, Ganote CE (1974) Structural changes in myocardium during acute ischemia. Circ Res 35(Suppl 3):156-172

2. Canty JM Jr, Suzuki G (2012) Myocardial perfusion and contraction in acute ischemia and chronic ischemic heart disease. J Mol Cell Cardiol 52:822-831

3. Reinstadler SJ, Thiele H, Eitel I (2015) Risk stratification by cardiac magnetic resonance imaging after ST-elevation myocardial infarction. Curr Opin Cardiol 30:681-689

4. Sengupta PP, Korinek J, Belohlavek M et al (2006) Left ventricular structure and function: basic science for cardiac imaging. J Am Coll Cardiol 48:1988-2001

5. Asgeirsson D, Hedstrom E, Jogi J et al (2017) Longitudinal shortening remains the principal component of left ventricular pumping in patients with chronic myocardial infarction even when the absolute atrioventricular plane displacement is decreased. BMC Cardiovasc Disord 17:208

6. Carlsson M, Ugander M, Heiberg E, Arheden H (2007) The quantitative relationship between longitudinal and radial function in left, right, and total heart pumping in humans. Am J Physiol Heart Circ Physiol 293:H636-H644

7. Carlsson M, Ugander M, Mosen H, Buhre T, Arheden H (2007) Atrioventricular plane displacement is the major contributor to left ventricular pumping in healthy adults, athletes, and patients with dilated cardiomyopathy. Am J Physiol Heart Circ Physiol 292: H1452-H1459

8. Hu K, Liu D, Herrmann S et al (2013) Clinical implication of mitral annular plane systolic excursion for patients with cardiovascular disease. Eur Heart J Cardiovasc Imaging 14:205-212

9. Romano S, Judd RM, Kim RJ et al (2017) Left ventricular long-axis function assessed with cardiac cine MR imaging is an independent predictor of all-cause mortality in patients with reduced ejection fraction: a multicenter study. Radiology. https:/doi.org/10.1148/ radiol.2017170529:170529

10. Rangarajan V, Chacko SJ, Romano S et al (2016) Left ventricular long axis function assessed during cine-cardiovascular magnetic 
resonance is an independent predictor of adverse cardiac events. $\mathrm{J}$ Cardiovasc Magn Reson 18:35

11. Ibanez B, James S, Agewall S et al (2018) 2017 ESC guidelines for the management of acute myocardial infarction in patients presenting with ST-segment elevation: the task force for the management of acute myocardial infarction in patients presenting with STsegment elevation of the European Society of Cardiology (ESC). Eur Heart J 39:119-177

12. Reindl M, Reinstadler SJ, Tiller C et al (2018) ACEF score adapted to ST-elevation myocardial infarction patients: the ACEF-STEMI score. Int J Cardiol 264:18-24

13. Reindl M, Reinstadler SJ, Tiller C et al (2019) Prognosis-based definition of left ventricular remodeling after ST-elevation myocardial infarction. Eur Radiol 29:2330-2339

14. Klug G, Metzler B (2013) Assessing myocardial recovery following ST-segment elevation myocardial infarction: short- and longterm perspectives using cardiovascular magnetic resonance. Expert Rev Cardiovasc Ther 11:203-219

15. Bondarenko O, Beek AM, Hofman MB et al (2005) Standardizing the definition of hyperenhancement in the quantitative assessment of infarct size and myocardial viability using delayed contrastenhanced CMR. J Cardiovasc Magn Reson 7:481-485

16. Mayr A, Klug G, Schocke M et al (2012) Late microvascular obstruction after acute myocardial infarction: relation with cardiac and inflammatory markers. Int J Cardiol 157:391-396

17. DeLong ER, DeLong DM, Clarke-Pearson DL (1988) Comparing the areas under two or more correlated receiver operating characteristic curves: a nonparametric approach. Biometrics 44:837-845

18. Ersboll M, Valeur N, Mogensen UM et al (2013) Prediction of allcause mortality and heart failure admissions from global left ventricular longitudinal strain in patients with acute myocardial infarction and preserved left ventricular ejection fraction. J Am Coll Cardiol 61:2365-2373

19. Bertini M, Ng AC, Antoni ML et al (2012) Global longitudinal strain predicts long-term survival in patients with chronic ischemic cardiomyopathy. Circ Cardiovasc Imaging 5:383-391

20. Zahid W, Johnson J, Westholm C et al (2013) Mitral annular displacement by Doppler tissue imaging may identify coronary occlusion and predict mortality in patients with non-ST-elevation myocardial infarction. J Am Soc Echocardiogr 26:875-884

21. Nielles-Vallespin S, Khalique Z, Ferreira PF et al (2017) Assessment of myocardial microstructural dynamics by in vivo diffusion tensor cardiac magnetic resonance. J Am Coll Cardiol 69:661-676

22. Wang Y, Cai W, Wang L, Xia R (2016) Evaluate the early changes of myocardial fibers in rhesus monkey during sub-acute stage of myocardial infarction using diffusion tensor magnetic resonance imaging. Magn Reson Imaging 34:391-396

23. Nguyen CT, Dawkins J, Bi X, Marban E, Li D (2018) Diffusion tensor cardiac magnetic resonance reveals exosomes from cardiosphere-derived cells preserve myocardial fiber architecture after myocardial infarction. JACC Basic Transl Sci 3:97-109

24. Brand B, Rydberg E, Ericsson G, Gudmundsson P, Willenheimer R (2002) Prognostication and risk stratification by assessment of left atrioventricular plane displacement in patients with myocardial infarction. Int J Cardiol 83:35-41

25. Sutherland GR, Di Salvo G, Claus P, D'Hooge J, Bijnens B (2004) Strain and strain rate imaging: a new clinical approach to quantifying regional myocardial function. J Am Soc Echocardiogr 17:788802

26. Kalam K, Otahal P, Marwick TH (2014) Prognostic implications of global LV dysfunction: a systematic review and meta-analysis of global longitudinal strain and ejection fraction. Heart 100:16731680

27. Pontone G, Guaricci AI, Andreini D et al (2017) Prognostic stratification of patients with ST-segment-elevation myocardial infarction (PROSPECT): a cardiac magnetic resonance study. Circ Cardiovasc Imaging 10

28. Yoon YE, Kang SH, Choi HM et al (2018) Prediction of infarct size and adverse cardiac outcomes by tissue tracking-cardiac magnetic resonance imaging in ST-segment elevation myocardial infarction. Eur Radiol 28:3454-3463

29. Romano S, Judd RM, Kim RJ et al (2018) Left ventricular long-axis function assessed with cardiac cine MR imaging is an independent predictor of all-cause mortality in patients with reduced ejection fraction: a multicenter study. Radiology 286:452-460

30. Garg P, Kidambi A, Swoboda PP et al (2017) The role of left ventricular deformation in the assessment of microvascular obstruction and intramyocardial haemorrhage. Int J Cardiovasc Imaging 33:361-370

31. Ochs MM, Fritz T, Andre F et al (2017) A comprehensive analysis of cardiac valve plane displacement in healthy adults: age-stratified normal values by cardiac magnetic resonance. Int $\mathrm{J}$ Cardiovasc Imaging 33:721-729

32. Pahlm U, Seemann F, Engblom H et al (2018) Longitudinal left ventricular function is globally depressed within a week of STEMI. Clin Physiol Funct Imaging. https://doi.org/10.1111/cpf. 12521

33. Rouleau JL, Talajic M, Sussex B et al (1996) Myocardial infarction patients in the 1990s-their risk factors, stratification and survival in Canada: the Canadian Assessment of Myocardial Infarction (CAMI) study. J Am Coll Cardiol 27:1119-1127

34. Reinstadler SJ, Klug G, Feistritzer HJ et al (2016) Prognostic value of left ventricular global function index in patients after ST-segment elevation myocardial infarction. Eur Heart J Cardiovasc Imaging $17: 169-176$

35. Eitel I, Poss J, Jobs A et al (2015) Left ventricular global function index assessed by cardiovascular magnetic resonance for the prediction of cardiovascular events in ST-elevation myocardial infarction. J Cardiovasc Magn Reson 17:62

36. van Kranenburg M, Magro M, Thiele $H$ et al (2014) Prognostic value of microvascular obstruction and infarct size, as measured by CMR in STEMI patients. JACC Cardiovasc Imaging 7:930-939

37. Niccoli G, Scalone G, Lerman A, Crea F (2016) Coronary microvascular obstruction in acute myocardial infarction. Eur Heart J 37: 1024-1033

38. de Waha S, Patel MR, Granger CB et al (2017) Relationship between microvascular obstruction and adverse events following primary percutaneous coronary intervention for ST-segment elevation myocardial infarction: an individual patient data pooled analysis from seven randomized trials. Eur Heart J 38:3502-3510

39. Klug G, Mayr A, Schenk S et al (2012) Prognostic value at 5 years of microvascular obstruction after acute myocardial infarction assessed by cardiovascular magnetic resonance. J Cardiovasc Magn Reson 14:46

40. Romano S, Judd RM, Kim RJ et al (2017) Association of featuretracking cardiac magnetic resonance imaging left ventricular global longitudinal strain with all-cause mortality in patients with reduced left ventricular ejection fraction. Circulation 135:2313-2315

41. Gavara J, Rodriguez-Palomares JF, Valente F et al (2017) Prognostic value of strain by tissue tracking cardiac magnetic resonance after ST-segment elevation myocardial infarction. JACC Cardiovasc Imaging. https://doi.org/10.1016/j.jcmg.2017.09.017

42. Stiermaier T, Lange T, Chiribiri A et al (2018) Left ventricular myocardial deformation in Takotsubo syndrome: a cardiovascular magnetic resonance myocardial feature tracking study. Eur Radiol 28:5160-5170

43. Luetkens JA, Schlesinger-Irsch U, Kuetting DL et al (2017) Feature-tracking myocardial strain analysis in acute myocarditis: diagnostic value and association with myocardial oedema. Eur Radiol 27:4661-4671 
44. Arenja N, Riffel JH, Halder M et al (2017) The prognostic value of right ventricular long axis strain in non-ischaemic dilated cardiomyopathies using standard cardiac magnetic resonance imaging. Eur Radiol 27:3913-3923

45. Eitel I, Stiermaier T, Lange T et al (2018) Cardiac magnetic resonance myocardial feature tracking for optimized prediction of cardiovascular events following myocardial infarction. JACC Cardiovasc Imaging. https://doi.org/10.1016/j.jcmg.2017.11.034

46. Barreiro-Perez M, Curione D, Symons R, Claus P, Voigt JU, Bogaert J (2018) Left ventricular global myocardial strain assessment comparing the reproducibility of four commercially available CMR-feature tracking algorithms. Eur Radiol 28:5137-5147

47. Hellwagner J, Wili N, Ibanez LF, Wittmann JJ, Meier BH, Ernst M (2018) Transient effects in pi-pulse sequences in MAS solid-state NMR. J Magn Reson 287:65-73

48. Lonborg JT, Engstrom T, Moller JE et al (2013) Left atrial volume and function in patients following ST elevation myocardial infarction and the association with clinical outcome: a cardiovascular magnetic resonance study. Eur Heart J Cardiovasc Imaging 14: $118-127$

49. Riffel JH, Andre F, Maertens M et al (2015) Fast assessment of long axis strain with standard cardiovascular magnetic resonance: a validation study of a novel parameter with reference values. J Cardiovasc Magn Reson 17:69

50. Gjesdal O, Yoneyama K, Mewton N et al (2016) Reduced long axis strain is associated with heart failure and cardiovascular events in the multi-ethnic study of atherosclerosis. J Magn Reson Imaging 44:178-185

Publisher's note Springer Nature remains neutral with regard to jurisdictional claims in published maps and institutional affiliations. 